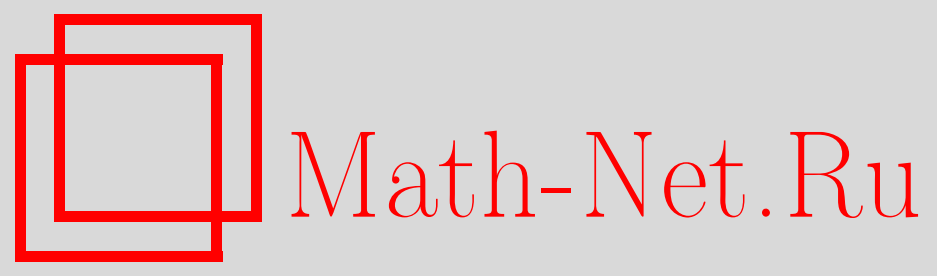

А. Н. Тимашёв, Закон больших чисел для перманентов случайных стохастических матриц, Дискрет. матем., 1999, том 11, выпуск 3, 91-98

DOI: https://doi.org/10.4213/dm389

Использование Общероссийского математического портала Math-Net.Ru подразумевает, что вы прочитали и согласны с пользовательским соглашением http://www.mathnet.ru/rus/agreement

Параметры загрузки:

IP: 3.91 .87 .62

26 апреля 2023 г., 13:08:39

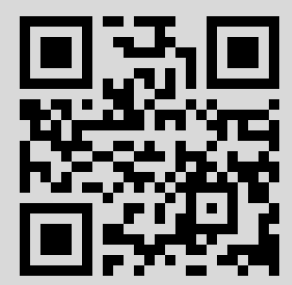


УДК 519.2

\title{
Закон больших чисел для перманентов случайных стохастических матриц
}

\author{
(C) 1999 г. А. Н. Тимашев
}

\begin{abstract}
Рассматривается класс всех квадратных $(0,1)$-матриц размера $n \times n$, имеющих в каждой строке $r$ единиц, $2 \leqslant r \leqslant n$. Для матрицы $P$, выбираемой случайно равновероятно из этого класса, получены достаточные условия асимптотического совпадения с вероятностью, стремящейся к единице, перманента per $P$ с его средним значением в схеме серий, когда при $n \rightarrow \infty$ параметр $r=r(n) \rightarrow \infty$ так, что $\sqrt{n}=o(r)$. Аналогичная задача решается для случайных стохастических матриц размера $n \times n$, строки которых являются независимыми в совокупности одинаково распределенными $n$-мерными случайными величинами, имеющими симметричное распределение Дирихле с параметром $\nu$ при условии, что при $n \rightarrow \infty$ параметр $\nu=\nu(n)>0$ меняется так, что $n \nu^{2} \rightarrow \infty$.
\end{abstract}

\section{1. Введение}

Рассмотрим класс всех квадратных $(0,1)$-матриц размера $n \times n$, имеющих в каждой строке $r$ единиц, $2 \leqslant r \leqslant n$. Число таких матриц равно $\left(\begin{array}{l}n \\ r\end{array}\right)^{n}$. Зададим на этом классе равновероятное распределение, приписав каждой матрице вероятность, равную $\left(\begin{array}{l}n \\ r\end{array}\right)^{-n}$. Пусть $P$ - матрица, случайно выбранная из этого класса. Рассмотрим случайную величину, равную перманенту per $P$. матрицы $P$.

Для подсчета математического ожидания $\mathbf{E} \operatorname{per} P$ и второго момента $\mathbf{E}(\operatorname{per} P)^{2}$ перманента матрицы $P$ используем метод, реализованный О'Нейлом ([1], стр. 7980). Упорядочим некоторым способом все подстановки симметрической группы $\sigma_{n}$ порядка $n$. Пусть $\sigma_{n}=\left\{s_{1}, s_{2}, \ldots, s_{n !}\right\}$ и $\Pi_{1}, \Pi_{2}, \ldots, \Pi_{n !}-$ соответствующие подстановочные матрицы. Положим $\xi_{i}=1$, если на местах, соответствущих единицам матрицы $\Pi_{i}$, в матрице $P$ стоят единицы, и $\xi_{i}=0$ в противном случае, $i=1,2, \ldots, n !$. Тогда

$$
\mathbf{P}\left\{\xi_{i}=1\right\}=\left(\frac{r}{n}\right)^{2}, \quad i=1,2, \ldots, n !,
$$

и поэтому из равенства

$$
\operatorname{per} P=\xi_{1}+\xi_{2}+\ldots+\xi_{n !}
$$

следует, что

$$
\text { E per } P=n !\left(\frac{r}{n}\right)^{2} \text {. }
$$


Далее,

$$
\begin{aligned}
\mathbf{E}(\operatorname{per} P)^{2} & =\mathbf{E} \operatorname{per} P+2 \sum_{1 \leqslant i<j \leqslant n !} \mathbf{P}\left\{\xi_{i}=\xi_{j}=1\right\} \\
& =n !\left(\frac{r}{n}\right)^{n}+2\left(\frac{r}{n}\right)^{n} \sum_{1 \leqslant i<j \leqslant n !}\left(\frac{r-1}{n-1}\right)^{n-l_{i j}},
\end{aligned}
$$

где $l_{i j}=n-\rho\left(s_{i}, s_{j}\right)$ при $i \neq j$ и

$$
\rho\left(s_{i}, s_{j}\right)=\operatorname{card}\left\{\nu \mid 1 \leqslant \nu \leqslant n ; s_{i}(\nu) \neq s_{j}(\nu)\right\}
$$

- расстояние между подстановками $s_{i}$ и $s_{j}, i, j=1,2, \ldots, n$.

Известно ([1], стр. 80), что $\rho\left(s_{i}, s_{j}\right) \geqslant 1$ при $i \neq j$ и поэтому $l_{i j} \leqslant n-1$; кроме того, для фиксированной подстановки $s_{i}$ число подстановок $s_{j}$ таких, что $\rho\left(s_{i}, s_{j}\right)=n-l$, выражается формулой

$$
\frac{n !}{l !} \sum_{m=0}^{n-l} \frac{(-1)^{m}}{m !}, \quad l=0,1, \ldots, n
$$

Отсюда получаем, что

$$
\begin{aligned}
\mathbf{E}(\operatorname{per} P)^{2} & =n !\left(\frac{r}{n}\right)^{n}+\left(\frac{r}{n}\right)^{n} \sum_{i=1}^{n !} \sum_{l=0}^{n !} \sum_{j: \rho\left(s_{i}, s_{j}\right)=n-l} \mathbf{P}\left\{\xi_{i}=\xi_{j}=1\right\} \\
& =n !\left(\frac{r}{n}\right)^{n} \sum_{m=0}^{n}(n)_{m}\left(\frac{r-1}{n-1}\right)^{m} S_{m},
\end{aligned}
$$

где

$$
S_{m}=\sum_{k=0}^{m} \frac{(-1)^{k}}{k !}, \quad m=0,1, \ldots
$$

И

$$
(n)_{0}=1, \quad(n)_{m}=n(n-1) \ldots,(n-m+1), \quad m=1,2, \ldots, n .
$$

Исходя из равенств (1), (2) и (3), в работе устанавливается закон больших чисел для перманента случайной матрицы $P$, аналогичный доказанным О'Нейлом законам больших чисел для перманентов случайных $(0,1)$-матриц с заданным числом единиц, а также с одинаковым числом единиц в каждой строке и каждом столбце $[2,3]$ (см. также [1], стр.79-85). Заметим, что если все элементы матрицы $P$ разделить на $r$, то получится случайная стохастическая матрица $P^{\prime}$, для которой per $P^{\prime}=r^{-n}$ per $P$, так что с учетом (1) и (2)

$$
\mathbf{E} \text { per } P^{\prime}=\frac{n !}{n^{n}}
$$

и

$$
\mathbf{E}\left(\operatorname{per} P^{\prime}\right)^{2}=\frac{n !}{r^{n} n^{n}} \sum_{m=0}^{n}(n)_{m}\left(\frac{r-1}{n-1}\right)^{m} S_{m} .
$$

Класс матриц вида $P$ рассматривается в [4], где сформулирован результат, аналогичный классической теореме Эрдеша-Реньи, оценивающий вероятность положительности перманента случайной $(0,1)$-матрицы с заданным числом единиц ([1], 
стр. 85). Таким образом, указанная аналогия распространяется и на закон больших чисел. Заметим, что актуальность рассматриваемой проблематики отмечалась в [5].

Пусть далее $C$ - случайная стохастическая матрица размера $n \times n$, строки которой являются независимыми в совокупности $n$-мерными одинаково распределенными случайными величинами, имеющими симметричное распределение Дирихле с параметром $\nu>0$. Плотность такого распределения имеет вид [6]

$$
p\left(x_{1}, \ldots, x_{n-1}\right)=\frac{\Gamma(n \nu)}{(\Gamma(\nu))^{n}} \prod_{j=1}^{n-1} x_{j}^{\nu-1}\left(1-\sum_{j=1}^{n-1} x_{j}\right)^{\nu-1}
$$

если $x_{j} \geqslant 0, j=1, \ldots, n-1$, и $x_{1}+\ldots+x_{n-1} \leqslant 1$, и $p\left(x_{1}, \ldots, x_{n-1}\right)=0$ в противном случае. Здесь $\Gamma-$ гамма-функция Эйлера.

Доказывается закон больших чисел для перманента per $C$ случайной стохастической матрицы $C$ при $n \rightarrow \infty$ в схеме серий, когда $\nu=\nu(n)>0$ и $n \nu^{2} \rightarrow \infty$. Заметим, что в случае $\nu=1$ в [7] показано, что с вероятностью единица $S(C) \rightarrow 1-\gamma$ при $n \rightarrow \infty$, где $S(C)$ - пропускная способность дискретного канала без памяти с матрицей переходных вероятностей $C$ и $\gamma=0,5772 \ldots$ - постоянная Эйлера.

\section{2. Дискретный случай}

Лемма 1. При $0<p \leqslant 1$ справедливо тождество

$$
\sum_{m=0}^{n}(n)_{m} S_{m} p^{m}=n ! p^{n} \sum_{m=0}^{n} \frac{\left(p^{-1}-1\right)^{m}}{m !}, \quad n=1,2, \ldots
$$

Доказательство. Положим

$$
\varphi_{n}(p)=\frac{1}{n !} \sum_{m=0}^{n}(n)_{m} S_{m} p^{m}, \quad n=0,1, \ldots,
$$

тогда

$$
\phi(z)=\sum_{n=0}^{\infty} \varphi_{n}(p) z^{n}=(1-p z)^{-1} \exp ((1-p) z)
$$

при $|z|<p^{-1}$. Пусть

$$
u(z)=(1-p z)^{-1}, \quad v(z)=\exp (1-p) z
$$

тогда согласно формуле Лейбница

$$
\phi^{(n)}(z)=\sum_{m=0}^{n}\left(\begin{array}{l}
n \\
m
\end{array}\right) u^{(m)}(z) v^{(n-m)}(z) .
$$

Поскольку

$$
u^{(m)}(z)=m ! p^{m}(1-p z)^{-(m+1)}
$$

и

$$
v^{(n-m)}(z)=(1-p)^{n-m} \exp ((1-p) z)
$$


при $z=0$ получаем, что

$$
\phi^{(n)}(0)=\sum_{m=0}^{n}(n)_{m} S_{m} p^{m}=n ! p^{n} \sum_{m=0}^{n} \frac{\left(p^{-1}-1\right)^{m}}{m !}
$$

Лемма 1 доказана.

Лемма 2. Справедливо равенство

$$
\mathbf{E}(\operatorname{per} P)^{2}=(n !)^{2}\left(\frac{r(r-1)}{n(n-1)}\right)^{n} \sum_{m=0}^{n} \frac{1}{m !}\left(\frac{n-r}{r-1}\right)^{m}, \quad r=2,3, \ldots, n .
$$

Доказательство. Положим $p=(r-1) /(n-1)$, тогда $0<p \leqslant 1$, если $2 \leqslant r \leqslant n$, и поэтому согласно (5) из (2) следует (6). Лемма 2 доказана.

Рассмотрим сначала случай, когда $r=2$. В этом случае при $n \rightarrow \infty$

$$
\mathbf{E}(\operatorname{per} P)^{2}=\frac{1}{2}(n !)^{2} n^{-2 n}\left(\frac{2 n}{n-1}\right)^{n} e^{n-2}(1+o(1))=\frac{\pi n}{e}\left(\frac{2}{e}\right)^{n}(1+o(1)) .
$$

Действительно, при $r=2$ согласно (6)

$$
\mathbf{E}(\operatorname{per} P)^{2}=(n !)^{2} n^{-2 n}\left(\frac{2 n}{n-1}\right)^{n} \sum_{m=0}^{n} \frac{(n-2)^{m}}{m !}
$$

Положим $\lambda=n-2, n \geqslant 3$, и пусть $\xi-$ случайная величина, распределенная по закону Пуассона с параметром $\lambda$. Тогда

$$
e^{-(n-2)} \sum_{m=0}^{n} \frac{(n-2)^{m}}{m !}=\mathbf{P}\{\xi \leqslant \lambda+2\}=\mathbf{P}\left\{\frac{\xi-\lambda}{\sqrt{\lambda}} \leqslant \frac{2}{\sqrt{\lambda}}\right\}=\frac{1}{2}+o(1)
$$

при $\lambda \rightarrow \infty$. Отсюда при $r=2$ получаем формулу для $\mathbf{E}(\operatorname{per} P)^{2}$, приведенную выше.

Лемма 3. Прu $n \rightarrow \infty u 3 \leqslant r \leqslant n$

$$
\mathbf{E}(\operatorname{per} P)^{2}=(n !)^{2} n^{-2 n}(r(r-1))^{n} \exp ((n-1) /(r-1))(1+o(1)) \text {. }
$$

Доказательство. При $r=n$ равенство (7), очевидно, выполняется. Пусть $3 \leqslant r<n$, тогда $\lambda=(n-r) /(r-1)>0$. Если $\xi-$ случайная величина, распределенная по закону Пуассона с параметром $\lambda$, то

$$
\begin{aligned}
\exp \left(-\frac{n-r}{r-1}\right) \sum_{m=0}^{n} \frac{1}{m !}\left(\frac{n-r}{r-1}\right)^{m} & =\mathbf{P}\{\xi \leqslant(r-1) \lambda+r\} \\
& =\mathbf{P}\{(\xi-\lambda) / \sqrt{\lambda} \leqslant(r-2) \sqrt{\lambda}+r / \sqrt{\lambda}\}
\end{aligned}
$$

Предположим сначала, что $r=o(n)$ при $n \rightarrow \infty$, тогда $\lambda \rightarrow \infty$ при $n \rightarrow \infty$, поэтому при $r \geqslant 3$

$$
\mathbf{P}\{(\xi-\lambda) / \sqrt{\lambda} \leqslant(r-2) \sqrt{\lambda}+r / \sqrt{\lambda}\}=1+o(1) .
$$


Теперь предположим, что $r \rightarrow \infty$ при $n \rightarrow \infty$, тогда в силу неравенства Маркова для неотрицательной случайной величины $\xi$

$$
\mathbf{P}\{\xi \leqslant n\} \geqslant 1-\frac{\lambda}{n}=1-\frac{n-r}{n(r-1)}=1+o(1) .
$$

Таким образом, при условиях леммы $3 \mathbf{P}\{\xi \leqslant n\}=1+o(1)$, и поэтому из (6) следует (7). Лемма 3 доказана.

Теорема 1. Пусть $n \rightarrow \infty u r=r(n) \rightarrow \infty$ maк, что $\sqrt{n}=o(r)$. Тогда последовательность случайных величин $\eta_{n}=\operatorname{per} P /$ Eer $P$ сходится по вероятности $\kappa$ единице.

Доказательство. Ясно, что

$$
\eta_{n}=\frac{\operatorname{per} P}{\mathrm{E} \operatorname{per} P}=\frac{1}{n !}\left(\frac{n}{r}\right)^{n} \operatorname{per} P
$$

при $n \rightarrow \infty$ и $r \geqslant 3$ согласно (7)

$$
\mathbf{E} \eta_{n}^{2}=\left(1-\frac{1}{r}\right)^{n} \exp ((n-1) /(r-1))(1+o(1))
$$

Если $\sqrt{n}=o(r)$, то

$$
n \ln n\left(1-\frac{1}{r}\right)+\frac{n-1}{r-1}=o(1)
$$

и поэтому

$$
\mathbf{E} \eta_{n}^{2}=1+o(1)
$$

Так как $\mathbf{E} \eta_{n}=1$, то $\mathbf{D} \eta_{n}=o(1)$, и если $\varepsilon>0$, то согласно неравенству Чебышева

$$
\mathbf{P}\left\{\left|\eta_{n}-1\right| \geqslant \varepsilon\right\} \leqslant \varepsilon^{-2} D \eta_{n} .
$$

Если положить

$$
\varepsilon=\varepsilon_{n}=\sqrt[3]{\mathrm{D} \eta_{n}}, \quad n=1,2, \ldots,
$$

то $\varepsilon_{n}>0$ при $3 \leqslant r<n$ и $\varepsilon_{n}=o(1)$ при $n \rightarrow \infty$, так что

$$
\varepsilon_{n}^{-2} \mathbf{D} \eta_{n}=\varepsilon_{n} \rightarrow 0
$$

и

$$
\lim _{n \rightarrow \infty} \mathbf{P}\left\{\left|\eta_{n}-1\right| \geqslant \varepsilon_{n}\right\}=0
$$

Теорема 1 доказана.

Замечание 1. Если $n \rightarrow \infty$ и $r^{2} / n \rightarrow \alpha \in(0, \infty)$, то

$$
\mathbf{E}(\operatorname{per} P)^{2}=(n !)^{2}\left(\frac{r}{n}\right)^{2 n} \exp \left(\frac{1}{2 \alpha}\right)(1+o(1)),
$$

поскольку в этом случае

$$
n \ln \left(1-\frac{1}{r}\right)+\frac{n-1}{r-1}=\frac{1}{2 \alpha}+o(1) .
$$

Если же $r=o(\sqrt{n})$ при $n \rightarrow \infty$, то $(\mathbf{E} \operatorname{per} P)^{2}=o\left(\mathbf{E}(\operatorname{per} P)^{2}\right)$ согласно (7). 
Замечание 2. Условие $\sqrt{n}=o(r)$ теоремы 1 означает, что $n^{3 / 2}=o(N)$, где $N=r n$ - число единиц в матрице $P$. Известно ([1], стр. 83), что для справедливости закона больших чисел для перманентов случайных $(0,1)$-матриц размера $n \times n$ с заданным числом $N$ единиц достаточно, чтобы $N>n^{3 / 2+\varepsilon}$, где $\varepsilon \in(0,1 / 2)$ и $n^{\varepsilon} \rightarrow \infty$ при $n \rightarrow \infty$. Это подтверждает указанную выше аналогию в формулировках законов больших чисел для случайной матрицы $P$, имеющей $r$ единиц в каждой строке, и случайной $(0,1)$-матрицы размера $n \times n$, содержащей $N=r n$ единиц.

\section{3. Непрерывный случай}

Пусть $C=\left\|c_{i j}\right\|$ - случайная стохастическая матрица размера $n \times n$, определенная выше. Распределение каждой строки этой матрицы совпадает с распределением случайного вектора $\left(\xi_{1}, \ldots, \xi_{n-1}, \xi_{n}\right)$, где

$$
\xi_{n}=1-\sum_{i=1}^{n-1} \xi_{i}
$$

и $(n-1)$-мерный случайный вектор $\left(\xi_{1}, \ldots, \xi_{n-1}\right)$ имеет плотность вида $(4)$.

Заметим, что (см. [6])

$$
\mathbf{E} \xi_{i}=\frac{1}{n}, \quad i=1,2, \ldots, n,
$$

и что в случае $\nu=1$ вектор $\left(\xi_{1}, \ldots, \xi_{n-1}\right)$ имеет равномерное распределение на $(n-1)$-мерном симпликсе

$$
\Omega_{n-1}=\left\{\left(x_{1}, \ldots, x_{n-1}\right) \mid x_{i} \geqslant 0 ; i=1, \ldots, n-1 ; x_{1}+\ldots+x_{n-1} \leqslant 1\right\}
$$

с плотностью $p\left(x_{1}, \ldots, x_{n-1}\right)=(n-1)$ !, если $\left(x_{1}, \ldots, x_{n-1}\right) \in \Omega_{n-1}$, и $p\left(x_{1}, \ldots, x_{n}\right)=$ 0 в противном случае. По определению

$$
\operatorname{per} C=\sum_{\left(i_{1}, \ldots, i_{n}\right)} c_{1 i_{1}} \ldots c_{n i_{n}}
$$

где суммирование проводится по всем $n$ ! перестановкам $\left(i_{1}, \ldots, i_{n}\right)$ чисел $1,2, \ldots, n$. Поскольку строки матрицы $C$ независимы в совокупности,

$$
\mathbf{E} \text { per } C=\sum_{\left(i_{1}, \ldots, i_{n}\right)} \mathbf{E} c_{1 i_{1}} \ldots \mathbf{E} c_{n i_{n}} .
$$

Из (9) и (10) следует, что

$$
\mathbf{E} \operatorname{per} C=\frac{n !}{n^{n}}
$$

При наших условиях все двумерные случайные векторы вида $\left(\xi_{i}, \xi_{j}\right), i \neq j, i, j=$ $1,2, \ldots, n$, имеют одинаковое распределение, и поэтому (см. [6])

$$
\mathbf{E}\left(\xi_{i} \xi_{j}\right)=\mathbf{E}\left(\xi_{1} \xi_{2}\right)=\frac{\nu}{n(n \nu+1)}, \quad i \neq j, \quad i, j=1,2, \ldots, n
$$

и

$$
\mathbf{E} \xi_{i}^{2}=\mathbf{E} \xi_{1}^{2}=\frac{\nu+1}{n(n \nu+1)}, \quad i=1,2, \ldots, n
$$


Лемма 4. Справедливо равенство

$$
\mathbf{E}(\operatorname{per} C)^{2}=\frac{n !}{n^{n}}\left(\frac{\nu+1}{n \nu+1}\right)^{n} \sum_{m=0}^{n}(n)_{m} S_{m}\left(\frac{\nu}{\nu+1}\right)^{m} .
$$

Доказательство. Проводя рассуждения, аналогичные использованным при обосновании формулы (2), получаем, что

$$
\mathbf{E}(\operatorname{per} C)^{2}=n ! \sum_{m=0}^{n}(n)_{m} S_{m}\left(\mathbf{E}\left(\xi_{1} \xi_{2}\right)\right)^{m}\left(\mathbf{E} \xi_{1}^{2}\right)^{n-m} .
$$

Подставляя в (13) значения $\mathbf{E}\left(\xi_{1} \xi_{2}\right)$ и $\mathbf{E} \xi_{1}^{2}$, приведенные выше, получаем (12). Лемма 4 доказана.

Лемма 5. Справедливо равенство

$$
\mathbf{E}(\operatorname{per} C)^{2}=\frac{(n !)^{2}}{n^{2 n}}\left(1-\frac{1}{1+n \nu}\right)^{n} \sum_{m=0}^{n} \frac{\nu^{-m}}{m !} .
$$

Доказательство. Формула (14) следует из (5) и (12), если положить $p=\nu /(\nu+1) \in$ $(0,1)$. Лемма 5 доказана.

Лемма 6. Пусть $n \rightarrow \infty u \nu=\nu(n)>0$ меняется так, ито $n \nu \rightarrow \infty$. Тогда

$$
\mathbf{E}(\operatorname{per} C)^{2}=\frac{(n !)^{2}}{n^{2 n}}\left(1-\frac{1}{1+n \nu}\right)^{n} \exp (1 / \nu)\left(1+O\left(\frac{1}{n \nu}\right)\right)
$$

Доказательство. Положим $\lambda=1 / \nu>0$, и пусть $\xi$ - случайная величина, распределенная по закону Пуассона с параметром $\lambda$. Тогда в силу неравенства Маркова для неотрицательной случайной величины $\xi$

$$
\sum_{m=0}^{n} \frac{\nu^{-m}}{m !}=\exp (1 / \nu) \mathbf{P}\{\xi \leqslant n\} \geqslant \exp (1 / \nu)\left(1-\frac{1}{n \nu}\right),
$$

и поэтому при условиях леммы 6

$$
\sum_{m=0}^{n} \frac{\nu^{-m}}{m !}=\exp (1 / \nu)\left(1+O\left(\frac{1}{n \nu}\right)\right)
$$

Формула (15) следует из (14) и (16). Лемма 6 доказана.

Теорема 2. Пусть $n \rightarrow \infty u \nu=\nu(n)>0$ меняется так, ито $n \nu^{2} \rightarrow \infty$. Тогда последовательность случайных величин $\theta_{n}=\operatorname{per} C / \mathrm{E} \operatorname{per} C$ сходится по вероятности к единице.

Доказательство. При условиях теоремы 2

$$
\left(1-\frac{1}{1+n \nu}\right)^{n}=\exp (-1 / \nu)\left(1+O\left(\frac{1}{n \nu^{2}}\right)\right)=\exp (-1 / \nu)(1+o(1))
$$

4 Дискретная математика, т.11 №3 
поскольку из условия $n \nu^{2} \rightarrow \infty$ следует, что $n \nu \rightarrow \infty$. Следовательно, с учетом (11) и (15)

$$
\mathbf{E}(\operatorname{per} C)^{2}=\frac{(n !)^{2}}{n^{2 n}}(1+o(1))=(\mathbf{E}(\operatorname{per} C))^{2}(1+o(1))
$$

и поэтому при $n \rightarrow \infty$

$$
\mathbf{D} \operatorname{per} C=o(\mathbf{E} \operatorname{per} C)^{2} \text {. }
$$

Полагая

$$
\theta_{n}=\frac{\operatorname{per} C}{\mathbf{E} \operatorname{per} C}=\frac{n^{n}}{n !} \operatorname{per} C,
$$

находим, что $\mathbf{E} \theta_{n}=1$ и $\mathbf{D} \theta_{n}=o(1)$ при $n \rightarrow \infty$.

Рассуждая далее так же, как при доказательстве теоремы 1 , получаем утверждение теоремы.

\section{Список литературы}

1. Сачков В. Н., Вероятностные методы в комбинаторном анализе. Наука, Москва, 1978.

2. O'Neil P., Asymptotic in random (0,1)-matrices. Proc. Amer. Math. Soc. (1970) 25, №2, 290-296.

3. O'Neil P., Asymptotic and random matrices with row-sum and column-sum restrictions. Bull. Amer. Math. Soc. (1969) 75, №6, 1276-1282.

4. Shevelev V. S., On projection of the Erdös-Renyi theorem about permanent of stochastic quadratic $(0,1)$-matrices into the subset of stochastic $(0,1)$-matrices with equal row sums. Третъя Всероссийскал икола-коллоквиум по стохастическим методам. Тезисъ докладов. ТВП, Москва, 1996, с. 177-178.

5. Сачков В. Н., Комбинаторные свойства неотрицательных матриц. Пробл. киберн. (1978) 26, 37-50.

6. Уилкс С., Математическал статистика. Наука, Москва, 1967.

7. Амбросимов А. С., Тимашев А. Н., Закон больших чисел для пропускной способности каналов без памяти со случайной переходной матрицей. Пробл. передачи информачии (1995) 31, №3, 24-34.

Статья поступила 07.05.1998. 